\title{
ON THE MECHANISM OF ATP-INDUCED SHAPE CHANGES IN HUMAN ERYTHROCYTE MEMBRANES
}

\author{
I. The Role of the Spectrin Complex
}

\author{
MICHAEL P. SHEETZ and S. J. SINGER \\ From the Department of Biology, University of California at San Diego, La Jolla, California 92093. \\ Dr. Sheetz's present address is the Department of Physiology, University of Connecticut School of \\ Medicine, Farmington, Connecticut 06032.
}

\begin{abstract}
Human erythrocyte ghosts have been shown, by scanning electron microscopy, to undergo ATP-dependent shape changes. Under appropriate conditions the ghosts prepared from normal disk-shaped intact cells adopt a highly crenated shape, which in the presence of $\mathrm{Mg}$-ATP at $37^{\circ} \mathrm{C}$ is slowly converted to the disk shape and eventually to the cup shape. These changes are not observed with other nucleotides or with 5'-adenylyl imidodiphosphate. Anti-spectrin antibodies, incorporated along with the Mg-ATP into the ghosts in amounts less than equivalent to the spectrin, markedly accelerate the shape changes observed with the Mg-ATP alone. The Fab fragments of these antibodies, however, have no effect. The conclusion is that the structural effect produced by the ATP is promoted by the cross-linking of spectrin by its antibodies, and may therefore itself be some kind of polymerization or network formation involving the spectrin complex on the cytoplasmic face of the membrane. The factors that contribute to the shape of the ghost and of the intact erythrocyte are discussed in the light of these findings.
\end{abstract}

It is widely assumed that the actomyosin-like proteins present in eukaryotic nonmuscle cells play a critical role in controlling cell shape and motility (for review see reference 15), but the molecular mechanisms for such control, and the involvement of the membrane in them, are not understood. The mechanochemical properties of the human erythrocyte are not only of great biomedical interest in themselves, but may serve as useful analogues for the related properties of more complex cells. The mature erythrocyte does have an actomyosin-like protein system, the spectrin complex, associated with its membrane (see Discussion), but it does not appear to have microtubules or other intracellular structural components to complicate the picture. Intact erythrocytes not only have a unique shape, but they undergo dramatic shape changes that are associated with changes in intracellular ATP levels (27). In this paper we demonstrate by scanning electron microscopy that erythrocyte ghosts undergo ATP-dependent shape changes that are analogous to those of intact cells, and we directly implicate the spectrin complex in these changes. With these observations, and the bilayer couple hypothesis (18), we attempt a more detailed analysis of erythrocyte shape changes than has heretofore been made. Some of these results have been presented in summary form else- 
where (20). In the following paper (2), further aspects of the biochemistry of these shape changes are presented.

\section{MATERIALS AND METHODS \\ Reagents}

ATP- $\gamma^{32}-\mathrm{P}$ and 5 '-adenylyl imidodiphosphate (AMPPNP) were obtained from ICN Pharmaceuticals Inc. (Cleveland, Ohio.). The former was diluted with sodium ATP from Calbiochem (San Diego, Calif.). Fresh human blood from healthy donors was drawn in acid citratedextrose and used within 2 to 4 days.

\section{Anti-Human Spectrin Antibody}

The antigen-affinity column purified antibody has been characterized elsewhere (21). The monovalent Fab fragment was made from the purified antibody by the method of Porter (16). The activity of the Fab fragments was assayed by their ability, when preincubated with spectrin, to prevent the precipitation of the spectrin by its bivalent antibody near the equivalence point in the antigen-antibody precipitation curve. In this manner, an amount of Fab that was 0.77 times the weight of bivalent antibody was taken as the equivalent of the intact antibody.

\section{Shape Changes of Ghosts}

Samples of fresh human blood were washed with 146

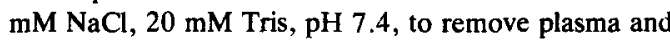
the buffy coat by centrifugation at $1500 \mathrm{~g}$ for $15 \mathrm{~min}$ The final pellet was diluted to $50 \%$ hematocrit and $1 \mathrm{vol}$

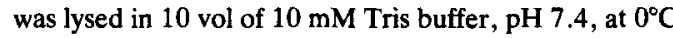
either with or without various additives (e.g., Mg-ATP and anti-spectrin antibody) present in different experiments. After $1 / 2 \mathrm{~h}$ incubation at $0^{\circ} \mathrm{C}$, the ghosts were pelleted at $27,000 \mathrm{~g}$ for $5 \mathrm{~min}$ and the supernate was drawn off. 9 vol of water containing the divalent cation and nucleotide at $0^{\circ} \mathrm{C}$ were added to 1 vol of the packed ghosts with mixing, and the ionic strength was raised to isotonic with the addition of 1 vol of $1.4 \mathrm{M} \mathrm{KCl}$, $0.2 \mathrm{M} \mathrm{NaCl}$, and $10 \mathrm{mM}$ Tris, $\mathrm{pH} 7.4$, at $0^{\circ} \mathrm{C}$. The ghosts were then brought to $37^{\circ} \mathrm{C}$ and samples were taken at various times and fixed by the addition of $1 \mathrm{vol}$ of ghost suspension to $0.5 \mathrm{vol}$ of $2 \%$ glutaraldehyde, $0.126 \mathrm{M} \mathrm{KCl}, 0.018 \mathrm{M} \mathrm{NaCl}, 10 \mathrm{mM}$ cacodylate, $\mathrm{pH}$ 7.4 , at $0^{\circ} \mathrm{C}$. After $15 \mathrm{~min}$ at $0^{\circ} \mathrm{C}$, an additional $0.5 \mathrm{vol}$ of fixative solution was added. $1 \mathrm{~h}$ later the ghosts were washed with distilled water by pelleting in a tabletop centrifuge and were post-fixed by $1 \% \mathrm{OSO}_{4}$ in distilled water for $20 \mathrm{~min}$ at $0^{\circ} \mathrm{C}$. Shapes of the ghosts were observed under the light microscope with phase contrast optics, and some samples were critical-point dried for viewing in the scanning electron microscope.

\section{Scanning Electron Microscopy}

After post-fixation with $1 \% \mathrm{OsO}_{4}$ the cells and ghosts were washed extensively with water, dehydrated with acetone, and critical-point dried with $\mathrm{CO}_{2}$ in a Bomar SPC-900 apparatus (The Bomar Co., Tacoma, Wash.). After rotary shadowing with Au-Pd, the cells were examined in an Etec model R1 scanning electron microscope.

\section{Incorporation of ${ }^{32} P$ into Spectrin \\ Band 2 upon Incubating \\ Ghosts with $\gamma^{32} P$-ATP}

Portions of the same samples of ghosts used in these experiments were used to study the kinetics of the shape changes. After lysis of erythrocytes (as in section 'Shape Change of Ghosts') and centrifugation of the ghosts, 1 vol of the pellet was diluted in $9 \mathrm{vol}$ of $2 \mathrm{mM} \gamma^{32}$ P-ATP $\left(0.05 \mathrm{C}_{\mathrm{l}} / \mathrm{mmol}\right)$ with or without $2 \mathrm{mM}$ divalent cation(either $\mathrm{Ca}$ or $\mathrm{Mg}$ ion), and then 1 vol of $1.4 \mathrm{M} \mathrm{KCl}, 0.2$

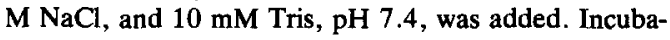
tion was stopped by denaturing and reducing samples with $2 \%$ SDS and $5 \% \beta$-mercaptoethanol at $100^{\circ} \mathrm{C}$. The samples containing approximately $50 \mu \mathrm{g}$ of membrane protein were applied to $0.1 \%$ SDS-polyacrylamide gels (3.25\% acrylamide) and were electrophoresed and stained according to the procedure of Fairbanks et al. (7). At the same time, three dilutions of a standard sample of purified spectrin were run for calibration of the protein concentration from the Coomassie blue staining intensities, as measured by a scanning densitometer trace (O.D. $550 \mu \mathrm{m}$ ). Stained gels were sliced in 2-mm slices and solubilized with $0.2 \mathrm{ml} 30 \% \mathrm{H}_{2} \mathrm{O}_{2}$ before counting with Aquasol (New England Nuclear, Boston, Mass.). Using the relative staining intensity of component 2 of spectrin and the number of counts of ${ }^{32} \mathrm{P}$ above background in that band of the stained gel, it was possible from the specific activity of the $\gamma^{32}$ P-ATP to calculate the moles of ${ }^{32} \mathrm{P}$ incorporated per mole of spectrin component 2, assuming that any ATP exchange was insufficient to change its specific activity.

\section{RESULTS}

\section{Shape Changes of Ghosts}

The intact normal human erythrocyte which is depleted of ATP assumes a crenated form and requires resynthesis of ATP to change back to a biconcave disk (27). By scanning electron microscopy, we have now found evidence that normal human erythrocyte ghosts undergo similar changes in shape. In isotonic salt solutions the ghosts assume a crenated form, but incubation with $\mathrm{Mg}$ ATP at $37^{\circ} \mathrm{C}$ induces a change to a shape which, except for smaller surface irregularities, closely approximates the biconcave disk form. On further incubation, cup-shaped ghosts form.

The ghost in a hypotonic solution initially assumed a spherical shape (not shown, see reference 20) but became crenated (Fig. $1 a$ ) when the ionic 

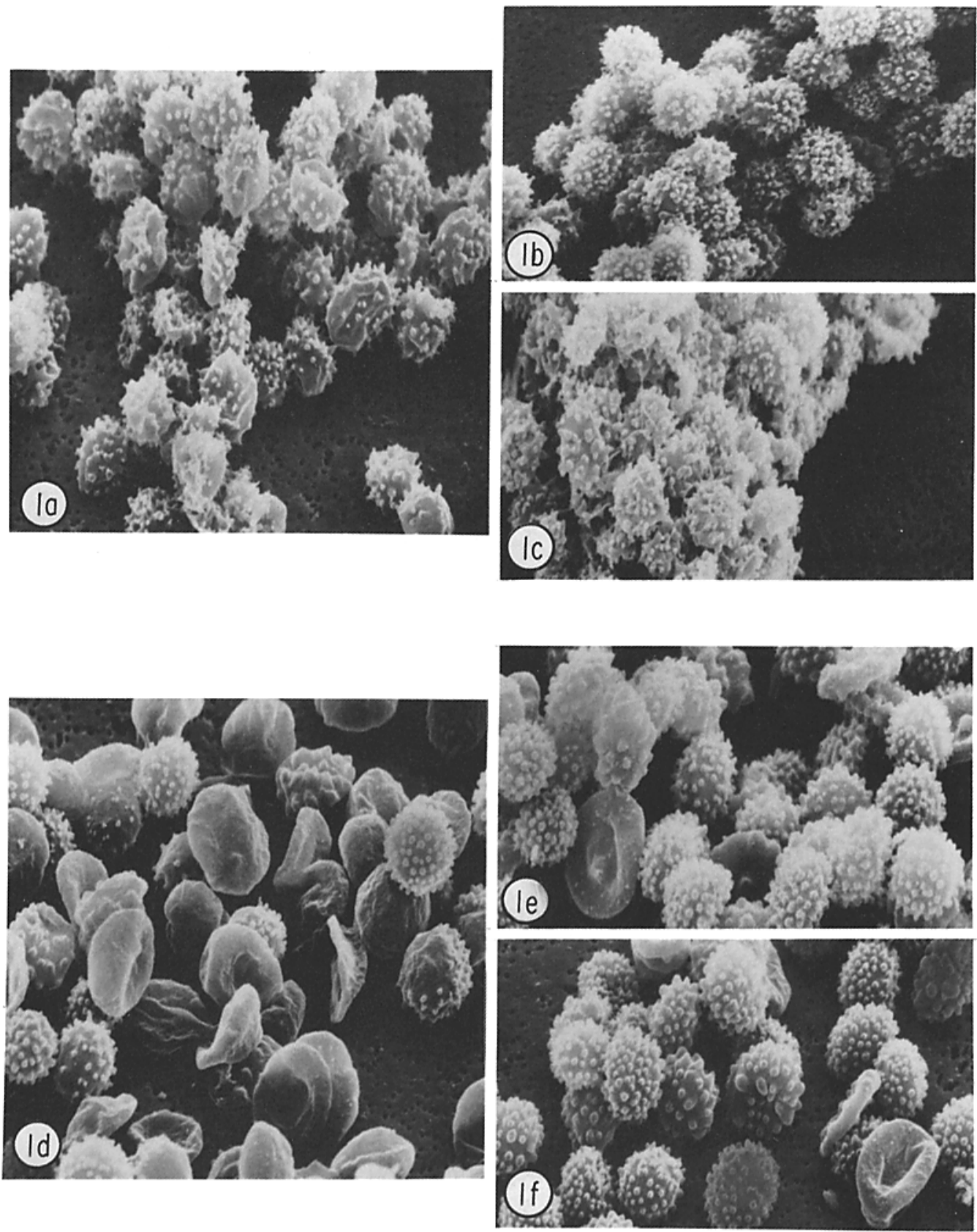

FIGURE 1 Scanning electron micrographs of normal human erythrocyte ghosts brought to isotonic ionic

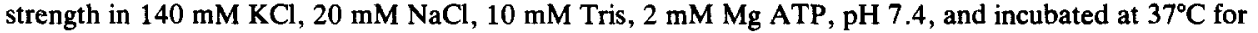
the indicated times. One sample, without any additional additives, was incubated for $0 \mathrm{~min}(b) ; 10 \mathrm{~min}(e)$; and $20 \mathrm{~min}(h)$. A second sample, that had been treated with $0.065 \mathrm{mg} / \mathrm{ml}$ of anti-spectrin antibody during the initial lysis of the cells, is shown after incubation for $0 \mathrm{~min}(a) ; 10 \mathrm{~min}(d) ; 20 \mathrm{~min}(g)$. A third sample, that was similarly treated with $0.05 \mathrm{mg} / \mathrm{ml}$ of the Fab fragments of the anti-spectrin antibody, is shown after incubation $0 \mathrm{~min}(c) ; 10 \mathrm{~min}(f) ; 20 \mathrm{~min}(i)$. The specimens $(h)$ and $(i)$ are indistinguishable, showing partial conversion of the ghosts to the biconcave disc shape, whereas specimen $(g)$ shows most of the ghosts converted. 

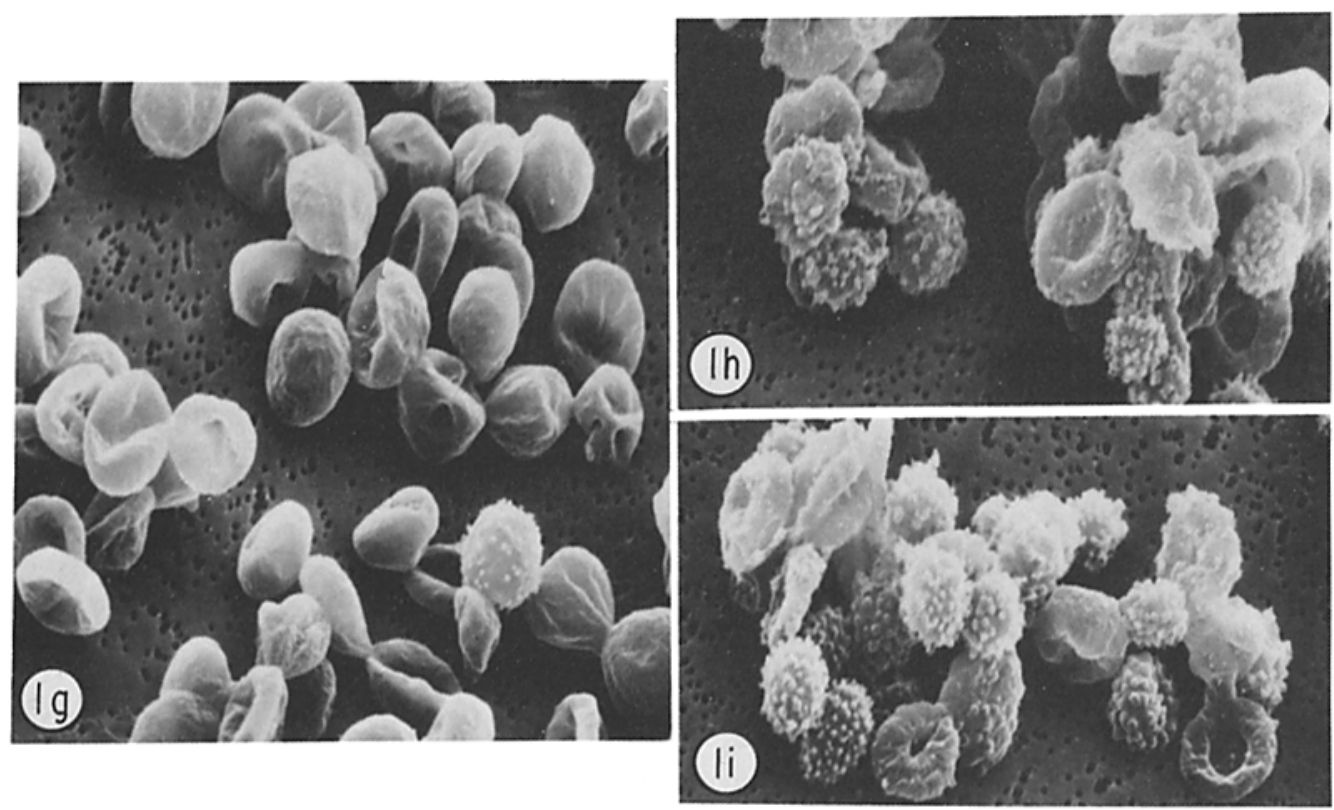

strength was raised to isotonic $(0.14 \mathrm{M} \mathrm{KCl}, 0.02$ $\mathrm{M} \mathrm{NaCl}$ ). This crenation occurred quickly even in the cold. When Mg-ATP (0.4-2 mM) was added to the ghosts before raising the ionic strength and the membranes were subsequently incubated at $37^{\circ} \mathrm{C}$ over a period of $30 \mathrm{~min}$, an increasing fraction of the ghosts was converted from the crenated form to biconcave disks (Fig. $1 h$ ). With further incubation at $37^{\circ} \mathrm{C}$, the membranes assumed an invaginated or cupped shape (not shown). At any time during this incubation, those ghosts that were still crenated had the same morphology as those at the start of incubation.

The transformation from crenated to disk to cupped shape could not be effected with ATP in the absence of $\mathrm{Mg}$ ion, nor with $\mathrm{Ca}-\mathrm{ATP}, \mathrm{Mg}-$ AMP-PNP, Mg-GTP, Mg-ITP, or Mg-CTP at the same concentrations. Furthermore, $10^{-3} \mathrm{M} \mathrm{Ca}$ ion along with $1 \mathrm{mM} \mathrm{Mg-ATP}$ completely inhibited the shape changes (Fig. 2); as little as $10^{-5} \mathrm{M} \mathrm{Ca}$ ion significantly inhibited them. The capacity to undergo the ATP-dependent shape changes was gradually lost during incubation at $0^{\circ} \mathrm{C}$ in hypotonic solutions, and was not observed with hemoglobin-free ghosts prepared according to Dodge et al. (5).

\section{The Effect of Anti-Spectrin Antibody} on the ATP-Dependent Shape Changes

If the spectrin complex plays a role in the ATP.

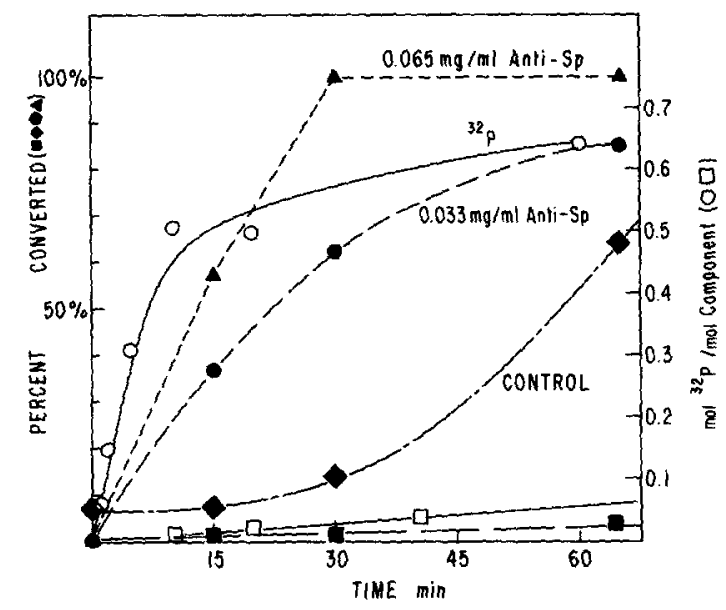

Figure 2 A comparison of the rates of the shape change to biconcave disk (filled data points) with the incorporation of ${ }^{32} \mathrm{P}$-phosphoryl groups from ATP by component 2 (open data points). The shape change is plotted for no additives $(\bullet)$ labeled "Control"; in the presence of $1 \mathrm{mM} \mathrm{Ca}^{++}(\mathbf{D})$; and in the presence of the indicated amounts of anti-spectrin antibody $(0,4)$. The ${ }^{32} \mathrm{P}$ incorporation is shown for the Control ghosts (O) and in the presence of $1 \mathrm{mM} \mathrm{Ca}^{++}(\square)$.

dependent shape changes, we reasoned that antispectrin antibody might affect the changes. Erythrocyte ghosts become impermeable to proteins almost immediately after lysis in hypotonic media; therefore, we lysed the erythrocytes directly into 
the $10 \mathrm{mM}$ Tris buffer containing the anti-spectrin antibody. Complement fixation studies of the antibody binding (21) indicated that lysis as described above in $0.5 \mathrm{mg} / \mathrm{ml}$ antibody solutions in the 10 $\mathrm{mM}$ Tris buffer corresponded to the spectrin-antibody equivalence point. Under these conditions, the membranes assumed the usual initial spherical shape, but remained so when the solution was made isotonic or when $\mathrm{Mg}$-ATP was present and incubation was carried out at $37^{\circ} \mathrm{C}$. By contrast, when the cells were lysed in $0.5 \mathrm{mg} / \mathrm{ml}$ of the monovalent Fab fragments of the antibody, the ghosts crenated upon the addition of salt and changed to biconcave disks with $\mathrm{Mg}-\mathrm{ATP}$ at $37^{\circ} \mathrm{C}$. On the other hand, if the concentration of bivalent antibody in the lysis buffer was lowered 10-20 fold, which resulted in the inclusion of one antibody molecule for every 5-10 spectrin molecules in the ghost, the ghosts did crenate and did transform to the biconcave disk shape with Mg-ATP present. However, when compared with control ghosts, such a population of bivalent antibodytreated ghosts changed shape at a much faster rate (compare the sequence Figure $1 a, d$, and $g$ with Fig. $1 b, e$, and $h$ ). As is seen in Fig. 2, without antibody there is normally a lag period at $37^{\circ} \mathrm{C}$ of about 10-20 min before significant numbers of biconcave disk ghosts are observed, and this lag period is very much shortened by the addition of the bivalent antibody. The Fab fragments of the anti-spectrin antibodies at equivalent concentration have no influence on the rate of the shape change (compare Fig. $1 b, e$, and $h$ with Fig. $1 c, f$, and $i$ ).

The results recorded in Fig. 2 were all obtained with a single preparation of ghosts to render them comparable. The percent conversion was determined by counting about 300 ghosts for each point on the graph. From one ghost preparation to the next the absolute positions of these kinetic curves varied, but their positions relative to one another remained closely similar.

\section{Phosphorylation of Spectrin}

We wanted to determine whether the known phosphorylation of spectrin component $2(28,1)$ that occurs upon adding $\gamma^{32} \mathrm{P}$-ATP to erythrocyte ghosts was correlated kinetically with the shape change. First, we found that under the conditions of the shape change experiments, component 2 was the only major protein component that acquired ${ }^{32} \mathrm{P}$ label (2). Second, the ionic requirements of the shape change and the phosphoryla- tion were closely similar. For example, no phosphorylation of spectrin occurred with ATP in the absence of $\mathrm{Mg}$ ion or in the presence of $\mathrm{Ca}$ ion, conditions under which the shape change did not occur either.

Phosphorylation of component 2 with $2 \mathrm{mM}$ $\mathrm{Mg}$-ATP occurred rapidly and reached a steady state well before the shape change was observed (Fig. 2). The kinetics of phosphorylation suggest a phosphate exchange on component 2 (2). At steady state, the specific activity of component 2 corresponded to $0.5 \mathrm{~mol}$ phosphoryl per mole protein. The presence of anti-spectrin antibody did not detectably alter the kinetics or the steadystate level of phosphorylation of component 2 .

\section{DISCUSSION}

In this paper, we report the occurrence of ATPdependent shape changes of human erythrocyte ghosts as observed by scanning electron microscopy. ${ }^{1}$ When biconcave disk-shaped intact cells are lysed into hypotonic buffer at $0^{\circ} \mathrm{C}$, and the ghosts are then brought to isotonic ionic strength at that temperature, the ghosts acquire a highly crenated shape (Fig. 1a). The ghosts remain crenated when the temperature is raised to $37^{\circ} \mathrm{C}$, and retain this shape indefinitely in the absence of added $\mathrm{Mg}$ ATP. Why these ghosts are crenated instead of being biconcave disks, like the intact cells from which they were derived, is not entirely clear, and is a problem discussed further below. If, however, $\mathrm{Mg}$-ATP at appropriate concentrations is added to the medium in which the cells are lysed, then after the return to isotonic ionic strength and $37^{\circ} \mathrm{C}$ the population of ghosts changes over a period of time from the crenated to the biconcave disk shape, and, on further incubation, to a cupped shape. ${ }^{2}$ It is this sequence of ATP-mediated shape changes that is the focus of this paper.

\footnotetext{
${ }^{1}$ Penniston and Green (13) have reported ATP-mediated shape changes in erythrocyte ghosts, but the observations were made by transmission electron microscopy of thin sections and under conditions different from ours, so the relevance of these earlier studies to ours and to the problem of erythrocyte shape is not clear. After this work was completed, Palek et al. (12) reported that incubation with $\mathrm{Mg}$-ATP induced erythrocyte ghosts to change from "flat discoidal" to "biconcave and cupshape" forms.

${ }^{2}$ At concentrations of $\mathrm{Mg}-\mathrm{ATP}>2 \mathrm{mM}$, after the change to cup shape, there further occurs an endocytosis of portions of the ghost membrane (Unpublished observations of W. Birchmeier and S. J. Singer).
} 
Although the population of ghosts shows a gradual change from crenated to disk shape, individual ghosts, once they start to change shape, appear to do so rapidly. That is, at any time after initiation of the shape change process, the population consists of two fractions: ( $i$ ) ghosts that have changed shape to biconcave disk and (ii) ghosts that are indistinguishable from the initial crenated population. One does not observe ghosts with fewer and fewer crenations in the course of the experiment, as one might expect if individual ghosts underwent a slow shape change. It thus appears that there is a lag period of different duration (between 10-60 min) for different individual ghosts, followed by a relatively rapid change in shape requiring a few minutes or less. The fact that limited amounts of anti-spectrin antibodies accelerate the shape change probably means that the antibodies act to shorten this lag period.

On the basis of the results in this and the following paper (2), together with other information to be cited, we can formulate a coherent molecular mechanism for these ATP-mediated ghost shape changes and, by analogy, for the similar ones which intact erythrocytes undergo. The four major elements involved in this mechanism are as follows:

\section{The Role of ATP}

The ghost shape changes from crenated to biconcave disk to cup shape have a specific requirement for Mg-ATP, no other nucleotide being effective. The $\gamma$-phosphate bond cleavage of ATP is necessary, since Mg-AMP-PNP is ineffective. On the other hand, AMP-PNP competes with ATP (2) and when present in excess inhibits the ATPinduced shape change. For reasons extensively discussed in the following paper (2), it is likely that the ATP acts as a substrate for an endogenous erythrocyte kinase rather than for an ATPase. (The ouabain analogue, strophanthidin, has no effect on the ATP-mediated shape changes; the $\mathrm{Na}$, $\mathrm{K}$-dependent ATPase (Na pump) is therefore not involved [2]). It is proposed that the kinase catalyzes a specific phosphorylation that is critical for the shape change to occur, and that this phosphorylation can be reversed by an endogenous phosphatase activity.

\section{The Role of the Spectrin Complex}

The spectrin complex consists of the two high molecular weight components 1 and 2 (collectively called spectrin), together with component 5 and perhaps others, which are peripherally attached to the cytoplasmic surface of the erythrocyte membrane (11). The complex is related to the actomyosin contractile proteins of muscle, because antibodies to human smooth muscle myosin crossreact with human spectrin (21), and component 5 is closely similar or identical to muscle actin (21, 25). We suggest that the function of ATP is to induce some kind of polymerization or network formation involving the spectrin complex on the membrane. This suggestion derives from the fact that less-than-equivalent amounts of anti-spectrin antibody incorporated into the ghosts markedly accelerated the ATP-mediated shape change, presumably by promoting the cross-linking of some of the spectrin. The lack of an effect of monovalent Fab fragments of the antibody indicates that it was the cross-linking of spectrin produced by the bivalent antibody, rather than the binding per se, that was responsible for the antibody effects. This synergistic effect of the antibodies and of ATP therefore suggests that the effect of the action of ATP itself is to induce some parallel kind of a polymerization or network formation involving the spectrin complex. On the other hand, larger amounts of antispectrin antibodies inhibited the ATP-induced shape changes, presumably by blocking the ATPinduced polymerization of the complex.

If such a polymerization is indeed induced by ATP, its detailed nature is not clear. It may involve not only the spectrin components to which the antibody was bound but component 5 (actin) and others as well. Some evidence for an interaction between erythrocyte spectrin and actin in vitro has been obtained (14).

On the other hand, it is also possible that the cross-linking of spectrin by its antibodies, and the effects produced by ATP, are structurally dissimilar, although synergistic, events.

\section{The Role of the Phosphorylation and \\ Dephosphorylation of Spectrin}

The indication that both an ATP-mediated phosphorylation (2) and a polymerization process involving spectrin are implicated in the ghost shape change focuses attention on the known phosphorylation of spectrin component 2 that occurs when $\mathrm{Mg}$-ATP is added to erythrocyte ghosts $(28,1)$. Spectrin component 2 is essentially the only protein component phosphorylated when the ATP-induced shape change occurs, and is the major (but not exclusive) phosphorylation product 
when normal intact erythrocytes are incubated with ${ }^{32} \mathrm{P}-\mathrm{P}_{1}(2)$. The actin component is not phosphorylated in either case. The ghost phosphorylation is rapid, and reaches a steady state well before the shape change is observed (Fig. 2). This would not be unreasonable if the polymerization on the membrane involving the already phosphorylated spectrin were the rate-limiting step in the shape change. When the ghosts are in the biconcave disk form, there is, on the average, less than one phosphoryl group per component 2 molecule. An endogenous slow dephosphorylation of component 2 that occurs (2) would allow the polymerization and the shape change to be reversible.

While the evidence is substantial (2) that the phosphorylation of component 2 is the chemical event by which ATP acts to effect the shape change, the evidence is still indirect, and it is not ruled out that some other as yet undetected event is involved.

\section{The Mechanics of the Shape Changes}

If we accept the hypothesis that an ATP-driven phosphorylation of component 2 leads to a polymerization or network formation involving the spectrin complex on the membrane, how does this process induce the shape change? Here we invoke the bilayer couple hypothesis $(18,19,22)$ to suggest an explanation. According to this hypothesis, the two half layers of an asymmetric bilayer membrane can respond differently to a perturbation of the membrane. For example, the inner half layer may contract its surface area relative to the outer half layer; if the membrane forms a closed surface, such an area change can lead to an evagination or crenation of the membrane. Conversely, an expansion of the inner surface area relative to the outer can lead to an invagination or cupping of the membrane. We propose, therefore, that the polymerization process involving the spectrin complex on the inner surface of the erythrocyte membrane leads to an expansion of that inner surface area relative to the outer; and the reverse process results in the opposite effects (22). There is some evidence that the spectrin complex is attached to the intramembranous particles where the latter protrude from the cytoplasmic surface of the erythrocyte membrane $(10,6)$. This attachment could provide a mechanism whereby the polymerization of the spectrin complex could generate the uniform two-dimensional tensions required for the expansion of the inner surface area.
It is premature, however, to explore such molecular mechanisms in detail at this time.

An important additional point is that this mode of attachment of the spectrin complex, together with its polymerization on the membrane, could explain the marked restriction of the mobilities of components in the intact erythrocyte membrane compared to the plasma membranes of other mammalian cells $(9,24)$. If each monomeric unit of the spectrin complex is attached to an intramembranous particle, then a polymer of the complex would effectively link together a large number of intramembranous particles, not only thereby immobilizing the latter in the plane of the membrane but also generating "picket-fence" barriers to the mobility of other integral components in the membrane.

When a ghost is prepared from a normal biconcave disk-shaped cell, we suggest that a combination of several factors including a detachment of some of the spectrin complex from the membrane (6), a mechanical disorganization of some of the remaining bound complex, together with some dephosphorylation of the complex (2), leads to a decrease in inner surface area and a crenation of the membrane in isotonic media. The ATP-mediated phosphorylation and proposed polymerization of the spectrin reverses these area changes. Hemoglobin-free ghosts prepared by the standard method of Dodge et al. (5) do not give the shape change but still contain considerable amounts of the spectrin complex. This suggests either that the bound spectrin complex must retain some organization which is disrupted upon extensive washing, or that the inner surface of the membrane has to be nearly saturated with the complex, or both, before the ATP-mediated shape change will occur.

These four elements comprise a mechanism for the ATP-mediated shape changes not only of ghosts but of intact erythrocytes. Storage of intact erythrocytes in unsupplemented serum or in isotonic buffers for $24 \mathrm{~h}$ at $37^{\circ} \mathrm{C}$ causes them to crenate. Accompanying the crenation is a marked decrease in intracellular ATP. If adenosine is then added to the medium, it is transported into the cells, the ATP is regenerated, and the cells are restored to the biconcave disk shape within $2 \mathrm{~h}$ at $37^{\circ} \mathrm{C}(27)$. These ATP and shape effects with the intact cells are therefore quite parallel to those we have observed with ghosts, and we infer that the same mechanisms are involved.

The mechanisms suggested in this and the fol- 
lowing paper (2) add some new perspectives to the long-standing enigma of erythrocyte shape. This is not the occasion to explore that problem in depth, but some relevant remarks are appropriate. The biconcave disk may be viewed as a steady-state shape which is under homeostatic control, with at least three considerations involved in that control.

(a) Previous work suggests that the volume of the cell is metabolically regulated to be smaller than that of a sphere of the same surface area (cf. 26). A critical factor in this regulation is thought to be the intracellular cation concentration, maintained by the active transport of $\mathrm{Na}$ and $\mathrm{K}$ ions by the $\mathrm{Na}, \mathrm{K}$-dependent ATPase in the cell membrane (26)

(b) While the maintenance of a reduced cell volume insures that the shape of the erythrocyte is nonspherical, it does not of itself determine that the shape be the biconcave disk. A given ratio of surface area to volume can be accommodated by any of a large variety of regular shapes including the biconcave disk (4). It has long been recognized that human erythrocyte ghosts can, under appropriate conditions, resume the biconcave disk shape (8), although an involvement of ATP in a change of ghosts to the biconcave disk shape as described in this paper has not been reported before. ${ }^{1}$ This indicates that structural features of the membrane itself in good part determine the biconcave disk shape. On the other hand, a static nonuniform structure in the plane of the membrane (i.e., the membrane in the regions of the dimples differing structurally from other regions of the membrane) cannot be responsible for the shape because the dimples can be relocated to different portions of the cell surface (3).

It has been suggested (4), however, that a membrane of uniform structure may spontaneously adopt the biconcave disk shape because it involves a minimum expenditure of bending energy compared to any other nonspherical shape. If this is correct, then it is very likely that it is the network formed by the spectrin complex attached to the membrane that is mainly responsible for the resistance to bending.

(c) A third factor involved in erythrocyte shape which has not been appreciated explicitly before arises from the results of the present and related studies. We suggest that it is not only the mean surface area of the cell but also the ratio, $r$, of inner and outer surface areas of the membrane (18) that is important to the biconcave disk shape. If $r$ is changed, even by less than 1\%, the shape of the cell is altered (18); the cell becomes crenated if $r$ decreases, and cupped if it increases. The value of $r$ is very likely influenced by many factors in vivo: to mention only a few possibilities, they include the exchange of lipids, fatty acids, and drugs with serum and serum components; endogenous fatty acyl transferase activity (23), which is confined to the inner half layer of the membrane (17); intracellular divalent cation concentrations; and the ATP concentration in the cell.

Homeostasis of the biconcave disk shape of the erythrocyte, in this view, would therefore involve not only the regulation of cell volume, but also the regulation of the area ratio $r$. Homeostasis of the area ratio might be achieved by adjusting the equilibrium extent of polymerization of the spectrin complex on the membrane, through changes in the intracellular free ATP concentration, or by other means.

M. P. Sheet was a Dernham Junior Fellow of the American Cancer Society, 1972-1974.

S. J. Singer is an American Cancer Society Research Professor.

This work was supported in part by U. S. Public Health Services grants GM-15971 and AI-06659.

Received for publication 3 September 1976, and in revised form 28 January 1977.

\section{REFERENCES}

1. Avruch, J., and G. Fairbanks. 1974. Phosphorylation of endogenous substrates by erythrocyte membrane protein kinases. I. A monovalent cationstimulated reaction. Biochemistry. 13:5507-5514

2. Birchmeier, W., and S. J. Singer. 1977. On the mechanism of ATP-induced shape changes in human erythrocyte membranes. II. The role of ATP. J. Cell Biol. 73:647-659.

3. Bull, B. 1973. Red cell biconcavity and deformability. In Red Cell Shape. M. Bessis, R. I. Weed, and P. F. Leblond, editors. Springer-Verlag, New York. 115-124.

4. Canham, P. B. 1970. The minimum energy of bending as a possible explanation of the biconcave shape of the human red blood cell. J. Theor. Biol. 26:6181.

5. Dodge, J. T., C. Mrtchell, and D. J. Hanahan. 1963. The preparation and chemical characteristics of hemoglobin-free ghosts of human erythrocytes. Arch. Biochem. Biophys. 100:119-130.

6. Elgsaeter, A., and D. Branton. 1974. Intramembrane particle aggregation in erythrocyte ghosts. I. The effects of protein removal. J. Cell Biol. 63:1018-1036. 
7. Fairbanks, G., T. Steck, and D. F. H. Wallach. 1971. Electrophoretic analysis of the major polypeptides of the human erythrocyte membrane. Biochemistry. 10:2606-2617.

8. Hoffman, J. F. 1958. Physiological characteristics of human red blood cell ghosts. J. Gen. Physiol. 42:9-28.

9. Loor, F., L. Forni, and B. Pernis. 1972. The dynamic state of the lymphocyte membrane. Factors affecting the distribution and turnover of surface immunoglobulins. Eur. J. Immunol. 2:203212.

10. Nicolson, G. L., and R. G. Painter. 1973. Anionic sites of human erythrocyte membranes. II. Antispectrin-induced transmembraue aggregation of the binding sites for positively charged colloidal particles. J. Cell Biol. 59:395-406.

11. Nicolson, G. L., V. T. Marchesi, and S. J. SINGER. 1971. The localization of spectrin on the inner surface of human red blood cell membranes by ferritin-conjugated antibodies. J. Cell Biol. 51:265-272.

12. Palek, J., G. Stewart, and F. J. Lionetti. 1974. The dependence of shape of human erythrocyte ghosts on calcium, magnesium, and ATP. Blood. 44:583-597.

13. Penniston, J. T., and D. E. Green, 1968. The conformational basis of energy transformations in membrane systems. IV. Energized states and pinocytosis in erythrocyte ghosts. Arch. Biochem. Biophys. 128:339-350.

14. Pinder, J. C., D. Bray, and W. B. Gratzer. 1975. Actin polymerization induced by spectrin. Nature (Lond.). 258:765-766.

15. Pollard, T. D., and R. R. Weining. 1974. Actin and myosin and cell movement. C.R.C. Crit. Rev. Biochem. 2:1-65.

16. Porter, R. R. 1959. The hydrolysis of rabbit $y$ globulin and antibodies with crystalline papain. Biochem. J. 73:119-126.

17. Renoois, W., L. M. G. Van Golde, R. F. A. ZwaAl, B. Roelofson, and L. L. M. Van DEENEN. Preferential incorporation of fatty acids at the inside of human erythrocyte membranes. Biochim. Biophys. Acta. 363:287-292.

18. Sheetz, M. P., and S. J. Singer. 1974. Biological membranes as bilayer couples. A molecular mechanism of drug-erythrocyte interactions. Proc. Natl. Acad. Sci. U. S. A. 71:4457-4461.

19. Sheetz, M. P., and S. J. Singer. 1976. Equilibrium and kinetic effects of drugs on the shapes of human erythrocytes. J. Cell Biol. 70:247-251.

20. Sheetz, M. P., R. G. Painter, and S. J. Singer. 1976. The contractile proteins of erythrocyte membranes and erythrocyte shapes changes. In Cell Motility. T. D. Pollard, J. Rosenbaum, editors. Cold Spring Harbor Conf. Cell Proliferation. 651-664.

21. Sheetz, M. P., R. G. Painter, and S. J. Singer. 1976. The relationships of the spectrin complex of human erythrocyte membranes to the actomyosins of muscle cells. Biochemistry. 15:4486-4492.

22. Sheetz, M. P., R. G. Painter, and S. J. Singer 1976. Biological membranes as bilayer couples. III. Compensatory shape changes induced in membranes. J. Cell Biol. 70:193-203.

23. Shohet, S. B., D. G. Nathan, and M. L. KarNovSKY. 1968. Stages in the incorporation of fatty acids into red blood cells. J. Clin. Invest. 47:10961108

24. Singer, S. J. 1974. Molecular biology of cellular membranes with applications to immunology. $A d v$. Immunol. 19:1-66.

25. Tilney, L. G., and P. Detmers. 1975. Actin in erythrocyte ghosts and its association with spectrin J. Cell Biol. 66:508-520.

26. Tosteson, D. C. 1964. Regulation of cell volume by sodium and potassium transport. In The Cellular Functions of Membrane Transport. J. F. Hoffman, editor. Prentice-Hall, Englewood Cliffs, N. J. 3-22.

27. Weed, R. I., P. L. La Celle, and E. W. MerRILl. 1969. Metabolic dependence of red cell deformability. J. Clin. Invest. 48:795-809.

28. Williams, R, O. 1972. The phosphorylation and isolation of two erythrocyte membrane proteins in vitro. Biochem. Biophys. Res. Commun. 47:671678. 